Cite as: Stephane H Maes, (2020), “Entanglement Concretizes Time in a Multi-fold Universe”, viXra:2010.0083v1, https://vixra.org/pdf/2010.0083v1.pdf, https://shmaesphysics.wordpress.com/2020/06/28/ entanglement-concretizes-time-in-a-multi-fold-universe/, June 28, 2020.

\title{
Entanglement and Random Walks Concretize Time in a Multi-fold Universe
}

\author{
Stephane H. Maes ${ }^{1}$
}

June 28, 2020

\begin{abstract}
:
In a multi-fold universe, gravity emerges from Entanglement through the multi-fold mechanisms. As a result, gravity-like effects appear in between entangled particles that they be real or virtual. Long range, massless gravity results from entanglement of massless virtual particles. Entanglement of massive virtual particles leads to massive gravity contributions at very smalls scales. Multi-folds mechanisms also result into a spacetime that is discrete, with a random walk fractal structure and non-commutative geometry that is Lorentz invariant and where spacetime nodes and particles can be modeled with microscopic black holes. All these recover General Relativity (GR) at large scales and semi-classical model remain valid till smaller scale than usually expected. Gravity can therefore be added to the Standard Model. This can contribute to resolving several open issues with the Standard Model.

The present paper examines what can be said of time in a multi-fold universe: what is the notion of time, does it exist or make sense and is it continuous or discrete and is there an arrow of time? In particular, we discuss how multi-fold universe handles the well-known time problem, the Bryce Wheeler equation as well as the explanations proposed so far by Page and Wootters and the subsequent rigorous expansions of Gambini and Pullin. In a multifold universe, time can concretely exist both because of entanglement and its random walk constructive nature that renders spacetime, including time discrete, fractal and non-commutative within a spacetime geometry, yet become not observable at larger scales. Therefore, random walks and entanglement concretize time and entanglement is also responsible for the arrow of time: the multi-folds mechanisms are irreversible, yet they can appear reversible if not fully modeled, which explains why Quantum Physics and GR appear essentially reversible. When putting all these consideration together it becomes clear that random walks and entanglement not only generate and shape spacetime but they also are at the core of the concept of time and how it can be perceived by us.
\end{abstract}

\section{Introduction}

The new preprint [1] proposes contributions to several open problems in physics like the reconciliation of General Relativity with Quantum Physics, explaining the origin of gravity proposed as emerging from quantum (EPREinstein Podolsky Rosen) entanglement between particles [5], detailing contributions to dark matter and dark energy and explaining other Standard Model mysteries without requiring New Physics beyond the Standard Model other than the addition of gravity to the Standard Model Lagrangian. All this is achieved in a multi-fold universe that may well model our real universe, which remains to be validated.

With the proposed model of [1], spacetime and Physics are modeled from Planck scales to quantum and macroscopic scales and semi classical approaches appear valid till very small scales. In [1], it is argued that spacetime is discrete, with a random walk-based fractal structure, fractional and noncommutative at, and above Planck scales (with a 2-D behavior and Lorentz invariance preserved by random walks till the early moments of the universe). Spacetime results from past random walks of particles. Spacetime locations and particles can be modeled as microscopic black holes (Schwarzschild for photons and spacetime coordinates, and metrics between

\footnotetext{
${ }^{1}$ shmaes.physics@gmail.com
} 
Reisner Nordstrom [2] and Kerr Newman [3] for massive and possibly charged particles - the latter being possibly extremal). Although surprising, [1] recovers results consistent with other like [4], while also being able to justify the initial assumptions of black holes from the gravity or entanglement model. The resulting gravity model recovers General Relativity (GR) at larger scale, as a 4-D process, with massless gravity, but also with massive gravity components at very small scale that make gravity significant these scales. Semi-classical models also work well till way smaller scales than usually expected.

In the present paper, we remain at a high level of analysis. It makes the points accessible to a wider audience and keeps the door open to further papers or discussions devoted to details of interest. Yet, it requires the reader to review [1], as we do not revisit here all the details of the multi-fold mechanism or reconstruction of spacetime. The followings subsections are organized as a series of observations in [1] as well as a few new considerations focused on what can be inferred about time at the level of the top down and bottom up phases of [1]. Our analysis is by no means exhaustive. However, we hope that it will intrigue enough the reader to push him or her to dig deeper. Most of the more detailed (or entry point) references are provided in [1], except for the new material, and so every statement is not motivated here or presented with the most appropriate references. This paper is rather a story tale. "[1]" appears often, as a person or a model, to refer to the original arguments, analysis, mechanisms or proposals discussed in [1].

\section{Entanglement effects in Multi-fold universes and Time Symmetry violations.}

The mechanisms of multi-folds, the main feature proposed in [1], trigger activation of additional structures (folds) when particles are (EPR) entangled so that additional paths can traverse the folds, where the EPR entangled particles can always meet as a same exit points. Doing so, all the activated folds (i.e. multi-folds) create attractive potentials in $\frac{1}{r}$ in between the entangled particles $\left(\frac{1}{r^{2}}\right.$ per fold). The attraction is towards their source or center the mass, depending of the use cases and movements (and masses involved - entangled particles can be massive or massless). When involving virtual particles emitted by a source of energy, this potential is reminiscent of gravity and [1] attributes gravity to these effects. It can also be looked as adding contributions of the effective Ricci curvature scalar $\mathrm{R}$ of the folds, from all matter or energy contributions, to build a new effective Ricci curvature scalar field $R$ and, with the direction of attraction information, a new consistent Ricci curvature tensor. Doing so, for all sources of energy, recovers Einstein's GR field equations (or Hilbert Einstein Action); which is amazing as invariance of surfaces (the real geometrical meaning behind the Hilbert Einstein Action) or variants of the Hilbert Einstein have, at no point, be postulated in [1] prior to that determination (something that can't exactly be said the same way for strings [28]). Also, the multi-folds have a spin-2 symmetry.

So, it is predicted in [1], that (EPR) entanglement between particles (or larger systems), results into attractive potentials in $\frac{1}{r}$ towards the center of mass, with $r$ the distance between form the center of mass, in between the entangled particles (on the support domain of the mapping), if integration takes place over $r$. That is over a system of entangled particles or for the range of uncertainty. Otherwise, each particles contribute a $\frac{1}{r^{2}}$ per fold contribution. For gravity, the integration of $r$ goes to infinity, hence the generic gravity like statement.

It is also important to note for completeness that [1] postulates that such effects only exist when entanglement is the result of interaction occurring locally (same source location). Other situations are considered as hierarchical and thought not to contribute an additional effective potential [28]. Yet, as in force composition, the different parts involved in a hierarchical event also amount to attractive effects; so attraction exist but as force composition. Also, if the entanglement is the effect of many repeated interactions (e.g. electron to phonon to electron), while hierarchical, the effects with composition will just appear as a normal non-hierarchical effect with attractive potential (at least in first approximation). So solid state entanglements a la superconductors for examples are modeled as nonhierarchical entanglement in this discussion; even if, in reality, it is the outcome of complex hierarchical composition of attractive potentials. 
The multi-fold mechanisms and mappings that are proposed in [1] are fundamentally time irreversible, for sure at the level of disentanglement, just as wavefunction collapse has also been conventionally presented as an irreversible process, yet more problematic because its model far from universally accepted. Here, disentanglement results into rupture of mapping and detachments of multi-folds from our spacetime: there is no way, yet envisaged in the context of [1], to reattach them or justification to try to do that, as in physical use cases, to try to do so; unless of course if achieving reversibility was the intent. It probably explains why conventional Quantum Physics can be modeled as emerging of quantum mechanics from classical irreversible thermodynamics $[29,30]$.

The evolution of the holographic mappings in [1] is intuitively motivated from the kinematics and dynamics of the multi-folds, as the result of "tearing them" out of spacetime post entanglement and following the entangled particles. Reversing time when this process is ongoing seems to mean magical reasons while the mapping exactly reverts, versus say staying where it was before reverting time or some other behavior not directly tracking the particle. Particles can revert their direction of movement to return to the entanglement location, but that is not time reversal. So, as shown in Figure 1, post time reversal, the mappings would seem to have a left over from where the entangled particles went before time reversal. The situation is therefore not symmetric. This is why [1] concluded that $T$ and $C P T$ symmetries are violated by the multi-fold mechanisms and therefore also by gravity that emerges from entanglement of virtual particles.

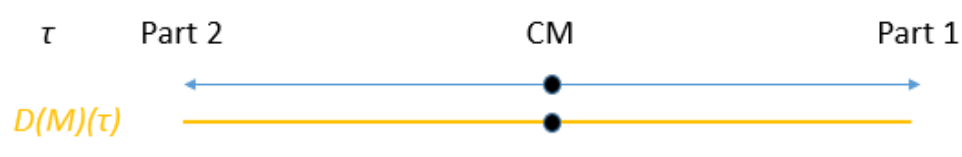

(a)

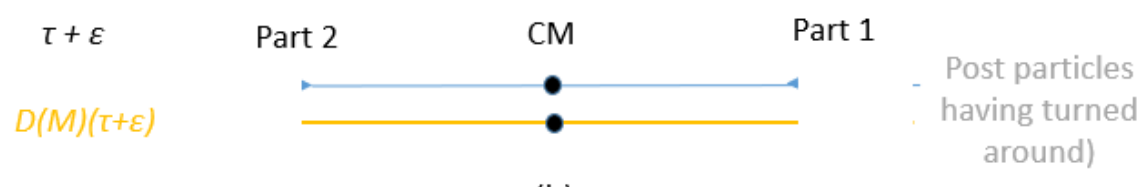

(b)

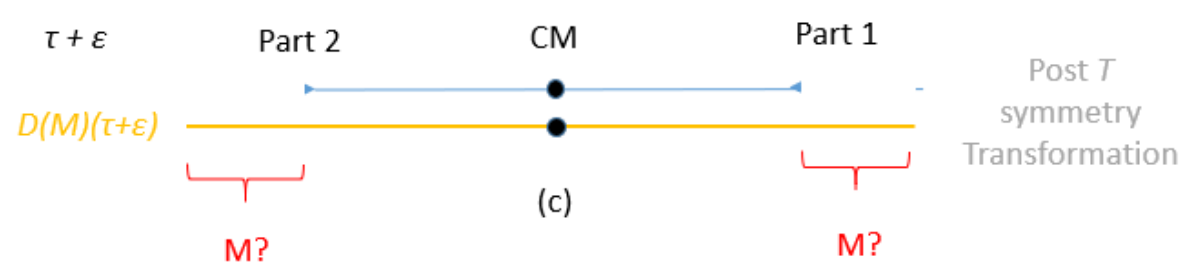

Figure 1: We can see the difference in terms of the support domain of the mappings involved in the multi-fold mechanisms of [1]. With time reversal, a portion of the domain mapping is undefined and has not reason to disappear. This leads to putative T symmetry violation and irreversibility of entanglement in multi-fold universe. In addition, disentanglement events in [1] are much more certainly irreversible.

\section{Reversible Physics?}

Yet, the fundamental laws of physics appear reversible in our real universe (with the considerations of [29,30]). Changing $t$ to $-t$ does not modify the laws of physics, that they be classical or quantum. It has been recently shown that quantum physics law can go back in time [6]. The second law of Thermodynamics can support statistical behaviors that typically supports irreversibility, albeit that interpretation in itself does not seem sufficient, or too ambiguous, for quantum processes [7,9]. 
Does this means that we have invalidated the suitability of [1] to model the real universe? We believe that it is to the contrary. The model of [1] seems to directly confirm Penrose conjecture [8] by providing an example of an irreversible law from which Quantum Physics and GR can emerge as reversible effective theories. Indeed, following [1], we see that both Quantum Field Theory (QFT) and GR do not model entanglement and / or track individual particles. From that point of view, they are statistical theories and in fact entanglement when modeled (only on the quantum side), is captured mainly via the entanglement entropy [31], and possible the modular Hamiltonian formalism (e.g. [32-35]). Without modeling entanglement and disentanglement, they lose the irreversibility aspects. It matters as, doing so, we may lose the ability to derive a problem free (e.g. divergence free and renormalizable) theory of quantum gravity using conventional QFT quantization techniques. In terms of quantum mechanics, able, by definition, to track particles and model particle entanglements, irreversibility is also lost as the wavefunction dynamics behavior when disentanglement takes place is not fully understood; just as for wavefunction collapses.

GR and Quantum Physics are doing a great job at modeling our world; without these concepts. So one can build effective reversible theories on irreversible foundations.

It is also worth noting as a digression that entangled particles have a lower entropy than disentangled ones. So an entangled system can be seen as a system away from equilibrium and its formation is consistent with irreversible thermodynamics because it is generated by a source or interaction (external system) that itself generated entropy in the production or interaction of the resulting entangled particles. Until further interactions or perturbation with something else take place, the particles stay in the entangled state of stationary superposition rather than (randomly) relaxing back into disentangled states. The whole process remains near equilibrium.

\section{Arrow of time}

The above indicates the nature of the evolutions to expect. Such an evolution has the potential to justify the arrow of time [10]: entanglement and disentanglement cannot be time reversed, especially disentanglement. As a result, system can only evolve according to the second law of Thermodynamics, even if all the other laws can be a priori reversed. Within a multi-fold universe, the cause for the arrow of time is microscopic; not just macroscopic (thermodynamic or cosmological). Of course, (dis)entanglement may or may not be the only cause for the arrow of time, depending, for example, where Physics ends up with its understanding of wave function and its collapse.

\section{The Problem of Time}

The above does not necessarily imply that a notion of time exists, just that a preferred direction of evolution of systems exists.

It has been argued that time itself may not exist, at least a concrete notion or observable [11,12]. The origin of the problem comes from a reformulation of GR with Hamiltonian in a foliated spacetime: the ADM formalism [13], where the Hamiltonian annihilates on the constraints to recover background independence. When used in a quantization program, the outcome is that the (field functional) wave function of the universe is stationary (as the Hamiltonian operator applied to it annihilates):

$$
H \psi=0
$$

(1) is known as the Wheeler-DeWitt equation [14] and it implies timelessness of the wave function. How can we make sense of this result, considering that the universe is full of non-stationary phenomena is what is called the problem of time [12]? Solving (1), is the original objective of Loop Quantum Gravity (LQG) [15]. Other models also aimed at addressing this. 
The paradox has been resolved or tentatively explained, by Page and Wootters, who observed that, within a closed system, internal observers (part of the system) can only refer to the evolution of observables or systems with respect to another internal observable [16]. On the other hand, for an external observer, no such "comparison" can take place as the entanglement of the "clock" and the rest of the physical system is in a stable but unknown superposition and therefore in a stationary state till it is measured; therefore explaining (1). A slightly more rigorous version, that amounts to the same physical explanation, as then reformulated by Pullin and Gambini [17]. [18], demonstrated experimentally these concepts.

[16-18] can be transposed to the multi-fold mechanisms of [1], based on our discussion above. The particles and activated multi-folds stay in a stationary state. Yes, the particles may move around, by the mechanisms follows them and rescales not caring at all about how it takes place as a time evolution. The effects are in $\frac{1}{r^{2}}$ per fold, no matter what and so it does not carry any temporal information to the outside; just the scale matters. No usable temporal information is available from AdS-5 (or the particles), not even their position as a way to measure unambiguously time. Therefore, we can safely state that [1] do not introduce concepts that would invalidate [1618]. In fact within a fold, time spent in the fold just disappears from the measure of the effective potential effect that depends only on the scale via $r$.

Furthermore, the conclusion also is that gravity, as derived from some (virtual pairs) entanglement, is stationary and does not carry temporal information; making it compatible, as should be by now, with (1). However, it would still be worth analyzing to analyze the reconstruction phase of [1] to see if more can be said. It is for future work.

\section{Discrete, Fractal, Non-commutative Time in Spacetime}

In [1], the multi-fold mechanisms imply that $\mathrm{AdS}(5)$ is non-commutative because spatial behaviors of the folds are linked to momentums. So is scale and therefore time. And so is our spacetime due to the mappings. Therefore, [1] predicts a discrete non-commutative spacetime, that is Lorentz invariant. Others have also demonstrated such discreteness and non-commutativity in the presence of quantum gravity $[19,20]$. [1] explains the Lorentz invariance by reconstruction via the random walk path also encountered in relativistic path integrals [22]. Randomness ensures the Lorentz invariance, captured in the non-commutative geometry and hence the existence of a minimum length to ensure no supra luminosity (at least for the reconstruction process of [1]). See also [36], for another kind of justification.

On this basis, [1] predicts a discrete, fractal, time part of the discrete spacetime. This means a minimum time length linked the minimum length by $c$. This is also consistent and predicted by other reasonings like [21], where it is shown that it also correspond to the minimum precision of clock synchronization ever achievable. Ord shows that a Fractal time is essential to recover the equations of Schrödinger, Klein Gordon and Dirac [22,23]. In any case, a continuous time, with a discrete or continuous space would not guarantee no supra luminosity (at least within the reconstruction process of [1]).

[1] also refers to [24] to show how both space and time fluctuations can be seen as responsible for particle and spacetime creation as processes contained in conventional physics.

The previous two paragraphs imply that time is a concrete parameter that exists, that it is discrete and, in fact, fractal. The discrete spacetime following non-commutative rules with space plays a key role in the spacetime Lorentz invariance.

But is time observable? To be observable, time would have to be encountered outside GR, conventional Quantum Physics and the multi-fold entanglement mechanisms. There is a fuzzy place where something observable happens: at very small scales, where spacetime location concretization or revisits occur: the apparition of new locations (in space hence observable) amounts to multiples of the fundamental minimum time length. Counting the clicks (of new locations concretized or revisited) amounts to counting evolution and it works because of the minimum temporal length. It is a variable available to hypothetical internal observers counting locally around its 
position. At larger scale it is not really observable, the moment that discreteness is not more noticeable: space point changes are not visible (because too small), nor are the clicks. Time become lost in Entanglement and only recoverable via clock entanglement with the system $[16,17]$. Time emerges from entanglement for inside observers.

What about external observers? We would argue that the process of counting discrete space locations and its evolution works the same for an external observer (locally or globally). So time is emergent and observable from the random walks. Again this effect is lost when discreteness of space(time) is not more visible, because at too small scales. Our result is not in contradiction with $[13,14,16,17]$ that does not model the discrete and random walk aspects. At larger scale, time is no more really observable the moment that discreteness is not more noticeable: spacetime point changes are not visible, nor are the clicks; time is lost in Entanglement and [16,17] shows us that we cannot entangle a clock as would be needed to externally observe time.

Internal observers can observe time at all scales. External can only observe when at scales where discreteness of spacetime is visible.

We believe this could be partially why LQG has challenges with deciding if it has or need an emergent time or not as mentioned in [25].

What about our macroscopic perception of time, where does it originate? [26] argues that it comes from noncommutativity and uncertainty because non-commutativity implies that order of event matters and order can be preserved and perceived at macroscopic level, where one can therefore pick certain events and their order to feel, infer and measure time. It is subjective. as linked to an observer reference frame, and we know that with Lorentz invariances, clock differ from reference frame to reference frame. Rovelli further ties this model to Thermal time derived by the Hamiltonian of the system (linked to time evolution via Schrödinger's equation. It is thermal because it would correspond to Boltzmann canonical states) as what is found out at quantum scales and above where the considerations can be statistical [27]. [1] derivation of non-commutativity and Lorentz invariance is compatible with such a view.

\section{Conclusions}

In this paper, we have how time appears in a multi-fold universe. We observed that the multi-fold mechanisms behind entanglement and gravity seem irreversible and break $T$ and CPT symmetries. It would imply the same for gravity [1].

We have shown compatibility with the notion that these mechanisms do not provide time as an observable to external observers, while maintaining the proposal that time can be determined by an observer part or entangled with the system.

We have shown that at very smalls scales i.e. around Planck scales, time is observable by all observers, but the notion becomes fuzzy and lost as we consider observations at larger scales. There we are compatible, through the underlying non-commutative Lorentz invariant spacetime, with a notion that time would result of order or flows of specific processes as observed by an internal observer while again remaining non observable for an external observer.

The novelty are really the irreversibility microscopic properties and T/CPT symmetry violations, already discussed in [1], and the observation that at very small-scale time will be observable by all, original to this paper. It is only when scale increases that it is lost and no more externally observable. At these scales, and above, we recover and are compatible with some (because the topic is controversial) of the conventional thinking. 
References: (most references come from popular science to make the discussion more approachable)

[1]: Stephane H. Maes, (2020) "Quantum Gravity Emergence from Entanglement in a Multi-Fold

Universe", viXra:2006.0088v1, (June 9, 2020).

[2]: https://en.wikipedia.org/wiki/Reissner\%E2\%80\%93Nordstr\%C3\%B6m metric

[3]: https://en.wikipedia.org/wiki/Kerr-Newman metric

[4]: Burinskii, Alexander, (2008), "The Dirac-Kerr-Newman electron", arXiv:0507109v4

[5]: https://en.wikipedia.org/wiki/EPR paradox

[6]: G. B. Lesovik, I. A. Sadovskyy, M. V. Suslov, A. V. Lebedev and V. M. Vinokur, (2019), "Arrow of time and its reversal on the IBM quantum computer", Scientific Reports volume 9, Article number: 4396

[7]: Kaonan Micadei, John P. S. Peterson, Alexandre M. Souza, Roberto S. Sarthour, Ivan S. Oliveira, Gabriel T. Landi, Tiago B. Batalhão, Roberto M. Serra, Eric Lutz, (2017), "Reversing the direction of heat flow using quantum correlations", arXiv:1711.03323v2

[8]: Roger Penrose, (1979), "Singularities and time asymmetry", in W. Israel and S. Hawking, "General relativity: an Einstein centenary survey", Cambridge

[9]: Brian Greene, (2004), "The Fabric of the Cosmos: Space, Time, and the Texture of Reality", Alfred Knopf

[10]: https://en.wikipedia.org/wiki/Arrow of time

[11]: https://en.wikipedia.org/wiki/Time in physics

[12]: https://en.wikipedia.org/wiki/Problem of time

[13]: https://en.wikipedia.org/wiki/ADM formalism

[14]: https://en.wikipedia.org/wiki/Wheeler\%E2\%80\%93DeWitt equation

[15]: https://en.wikipedia.org/wiki/Loop quantum gravity

[16]: Page, Don N.; Wootters, William K. (15 June 1983). "Evolution without evolution: Dynamics described by stationary observables". Phys. Rev. D. 27: 2885

[17]: Rodolfo Gambini, Rafael A. Porto, Jorge Pullin, and Sebastian Torterolo, (2009), "Conditional probabilities with Dirac observables and the problem of time in quantum gravity", Phys. Rev. D 79, 041501

[18]: Ekaterina Moreva, Giorgio Brida, Marco Gramegna, Vittorio Giovannetti, Lorenzo Maccone, Marco Genovese, (2014), "Time from quantum entanglement: an experimental illustration", arXiv:1310.4691v1

[19]: S. Doplicher, K. Fredenhagen and J. E. Roberts, (1994), "Spacetime quantization induced by classical gravity", Phys. Rev. B 331 (1994) 33.

[20]: Hooft, Gerard 't, (2016), "How quantization of gravity leads to a discrete space-time", J. Phys.: Conf. Ser. 701 012014

[21]: Sabine Hossenfelder, (2012), "Minimal Length Scale Scenarios for Quantum Gravity", arXiv:1203.6191v1

[22]: G N Ord, (1983), "Fractal space-time: a geometric analogue of relativistic quantum mechanics", J. Phys. A: Math. Gen. 16 (1983) 1869-1884.

[23]: G.N.Ord, (1996), "Fractal space-time and the statistical mechanics of random walks", Chaos, Solitons \& Fractals, Volume 7, Issue 6, June 1996, Pages 821-843

[24]: Hou Y. Yau, (2007 \& 2016), "Quantum Theory from a Space-Time Wave", arXiv:0706.0190 v2 and v4

[25]: Stephane H Maes, (2020), "Alignments and Gaps Between Multi-fold Universes And Loop Quantum

Gravity", viXra:2006.0229v1, https://shmaesphysics.wordpress.com/2020/06/19/multi-fold-universes-analysis-ofloop-quantum-gravity/, June 24, 2020.

[26]: Connes, A and Rovelli, C, (1994), "Von Neumann algebra automorphisms and time-thermodynamics relation in generally covariant quantum theories", Classical and Quantum Gravity. 11 (12): 2899-2917.

[27]: Carlo Rovelli, (2017), "The order of time", Penguin Books

[28]: Stephane H Maes, (2020), "Circular Arguments in String and Superstring Theory from a Multi-fold Universe

Perspective", https://shmaesphysics.wordpress.com/2020/10/11/circular-arguments-in-string-and-superstringtheory-from-a-multi-fold-universe-perspective/ , October 5, 2020.

[29]: D. Acosta, P. Fernandez de Cordoba, J. M. Isidro, J. L. G. Santander, (2012), “Emergent quantum mechanics as a classical, irreversible thermodynamics", arXiv:1206.4941v2

[30]: Stephane H Maes, (2020), "Implicit Multi-Fold Mechanisms in a Neural Network Model of the Universe", https://shmaesphysics.wordpress.com/2020/09/12/implicit-multi-fold-mechanisms-in-a-neuralnetwork-model-of-the-universe/, September 12, 2020. 
[31]: Daniel Cavalcanti Santos, (2008). "Entanglement: from its mathematical description to its experimental observation", PhD Thesis, Universitat de Barcelona, https://www.icfo.eu/images/publications/DT $0801 . p d f$. Retrieved on March 4, 2019.

[32]: J. J. Bisognano and E. H. Wichmann, (1976), "On the Duality Condition for Quantum Fields, " J. Math. Phys. 17, 303.

[33] P. D. Hislop and R. Longo, (1982), "Modular Structure of the Local Algebras Associated With the Free Massless Scalar Field Theory", Commun. Math. Phys. 84, 71 (1982).

[34] H. Casini, E. Teste and G. Torroba, (2017), "Modular Hamiltonians on the null plane and the Markov propertyof the vacuum state", J. Phys. A 50, no. 36, 364001.

[35]: Ted Jacobson, (2015), "Entanglement Equilibrium and the Einstein Equation", arXiv:1505.04753v4

[36]: Luis J Garay, (1995), "Quantum Gravity and minimum length", International Journal of Modern Physics A, Vol 10, N 2, 145-165

[37]: Ronald J. Adler, David I. Santiago, (1999), "On Gravity and the Uncertainty Principle", arXiv:gr-qc/9904026v2

[38]: Gao, Shan, (2013), "Three possible implications of spacetime discreteness", URL: http://philsciarchive.pitt.edu/id/eprint/9966. Retrieved on June 19, 2020. 